\title{
A cell for every season: the ovarian granulosa cell
}

\author{
David F. Albertini
}

Published online: 22 October 2011

(C) Springer Science+Business Media, LLC 2011

While those of us in the business of human ARTs tend to be preoccupied with gametes and embryos (the stars of the show), there is mounting awareness that behind the scenes are players without whose long term and supportive contributions, the matter of establishing pregnancy would be little more than an academic exercise. The supporting cast consists mainly of ovarian granulosa cells or testicular Sertoli cells. From the very beginnings of gonad development and throughout puberty, it has become apparent that granulosa or Sertoli cells are the drivers of sex determination and gatekeepers of the transition from adolescence to adulthood.

The drama unfolds deep within either the ovarian follicle or seminiferous tubule where their physical proximity to, and protracted interaction with, germ cells shape the very character of our gametes that allow them to generate a viable zygote.

These compartments provide a niche for their respective gametes that sustain, nurture and guide the complex process of gametogenesis over time intervals of weeks to months and all the while sharing a common purpose in the larger scheme of ARTs-healthy gametes. So, while Sertoli cells paternalistically engage their germ cells over a matter of weeks (before dismissing sperm to the more distal reaches of the epididymis), such is not the case for the granulosa cell. Instead, granulosa cells meld with oocytes as birth

Capsule Like the changing of the seasons, the ovarian granulosa cell must purposefully transform consonant with the evolving functions of the follicle. In so doing, the granulosa cell cyclically orchestrates the completion of oogenesis, ovulation, and formation of the corpus luteum.

D. F. Albertini $(\square)$

Kansas University Medical Center,

Kansas City, KS, USA

e-mail: dalbertini@kumc.edu partners within the primordial follicle holding out for as long as possible, decades for sure, before transitioning from a cold winter into spring (see below). It is this journey from primordial follicle to corpus luteum that makes the granulosa cell a chameleon for all seasons. It should come as no surprise then that the search for biomarkers of oocyte potential in the phenotype of the granulosa cell continues to be explored to identify those oocytes that make healthy pregnancies happen.

As efforts continue to focus on those qualities of an embryo that make it likely to produce a healthy child, more and more, attention is being drawn back into the depths of the gonad to understand what it is about the germ cell microenvironment or niche that makes a gamete what it is from the more neighborly, somatically speaking of course, point of view.

The present issue of JARG calls attention to two aspects of the biology of granulosa cells most pertinent to human ARTs. In the first case, work from the Pisarska laboratory extends in a novel way recent efforts to employ granulosa cell transcriptomics in the determination of human oocyte quality (see "Relative expression of genes encoding SMAD signal transduction factors in human granulosa cells is correlated with oocyte quality"). While TGF beta signaling has been at the forefront of follicle biology for more than a decade now, and the hunt for factors and their cognate receptors has continued in hopes of identifying relevant biomarkers, these studies are amongst the first to recognize the importance of systems biology in teasing apart the network of cell interactions that allow a granulosa cell to establish the developmental competence of its companion oocyte.

In the following (and neighboring) paper, Soukup and colleagues take us back to a time in the history of ovarian research when the importance and utility of culturing 
human granulosa cells was appreciated by experimentalists and clinicians alike (see "Proliferative potential and phenotypic analysis of long term cultivated human granulosa cells initiated by addition of follicular fluid"). As models for studying steroidogenesis and the mechanism of action of gonadotropins, cultured granulosa cells have been a mainstay in the field of ovarian physiology. Their role as sentinels of oocyte quality may have emerged more recently but points again to the extraordinary behavior that these cells exhibit at discrete stages of folliculogenesis. As interest grows in this area, the telltale signs of what a granulosa cell should be doing as the seasons change will improve both our understanding of follicle biology and potential treatment options for protecting the viability and health of oocytes intended for ARTs.

Reproductive medicine, as with many disciplines, has relied heavily on the use of hormones for altering physiology to obtain a therapeutically favorable outcome. Depending on the old adage, one hormone-one receptor, no longer is acceptable as the biochemical and pharmaceutical underpinnings of drug action inform us as to the shortcomings of a single target approach for manipulating physiological responsiveness. FSH is a good case in point. In today's world of signaling biology, it would be heretical to consider either the specificity of FSH as a modifier of gonadal physiology alone, given recent discoveries on its role in bone metabolism, or a single signaling pathway as responsible for driving follicular development and estrogen biosynthesis. Rather, there has been a gradual, albeit reluctant, change in attitude to examine disease or physiological processes as founded in many interacting networks of signaling pathways where multiple nodal intersects would be the subject of therapeutic intervention instead of a single target. To some, this may evoke a familiar response given the recent advances in studies on the mechanism of ovulation in mammals where LH emerges as just the tip of the iceberg!

It is in this new spirit of discovery that future studies on the granulosa cell will provide an experimental platform of tractable proportions for solving the deeper mysteries of the ovary and how it produces oocytes of clinical import. Searching for biochemical targets that modify the various potentialities of the granulosa cell as an ardent protector in the primordial follicle, as a source of nourishment and guidance during oocyte growth and differentiation, and finally as dualistic contributor to ovulation and luteinization, will pay off in the long run as small molecules assume their rightful role in the treatment and management of infertility. 